\title{
Increasing Isolation Between Colocated Antennas Using a Spatial Notch
}

\author{
Erwin Janssen, Member, IEEE, Dusan Milosevic, Matti Herben, Senior Member, IEEE, and \\ Peter Baltus, Member, IEEE
}

\begin{abstract}
This letter presents an antenna configuration to achieve a coupling reduction between colocated antennas. Application of an adaptive spatial notch enables this functionality. Analytical results are obtained, which show good resemblance to simulated and measured results, performed on a prototype manufactured to operate around $2.5 \mathrm{GHz}$. Coupling reduction of $50 \mathrm{~dB}$ has been measured. The antenna impedance is also influenced because of this configuration and shows an $S_{11}$ better than $-15 \mathrm{~dB}$ at the frequency of interest. In addition, the radiation pattern of both antennas is influenced. This can be seen as an advantage or a disadvantage, depending on the application.
\end{abstract}

Index Terms-Antenna isolation, antenna measurements, electromagnetic coupling, near field.

\section{INTRODUCTION}

I N MULTIMODE devices such as cell phones and PDAs, an increasing number of wireless standards are incorporated. Examples of this are the use of UMTS, WLAN, Bluetooth, FM radio, GPS, and GSM in a single device. The colocation of these different transceivers inherently leads to profound interference issues [1]. Present solutions to this problem can be subdivided into two categories, namely medium access (MAC) layer and physical (PHY) layer solutions.

Solving this issue in the MAC layer concentrates on attributing different time slots to the different standards [2], [3]. Hereby, the simultaneous operation of transmitters and receivers is circumvented. However, this may not always be possible, and leads to a reduced throughput. PHY-layer solutions rely on either coping with the strong interfering signal by increasing the linearity of the front end [4] or reducing the coupling between the transmitter and receiver [5]-[7]. Increasing the linearity of the front end leads to an increase of power consumption, an undesirable aspect in handheld devices. Reducing the coupling between the different standards enables simultaneous operation and leads to little additional cost in terms of power consumption.

In the literature, several approaches can be found to decrease the coupling between colocated antennas, and in many cases, there result tradeoffs between various aspects. For example,

Manuscript received February 11, 2011; revised March 27, 2011 and May 10, 2011; accepted May 20, 2011. Date of publication June 02, 2011; date of current version June 23, 2011. This work was supported by the Technology Foundation STW.

The authors are with the Department of Electrical Engineering, Eindhoven University of Technology, Eindhoven 5600 MB, The Netherlands (e-mail: E.J.G.Janssen@tue.nl).

Color versions of one or more of the figures in this letter are available online at http://ieeexplore.ieee.org.

Digital Object Identifier 10.1109/LAWP.2011.2158510

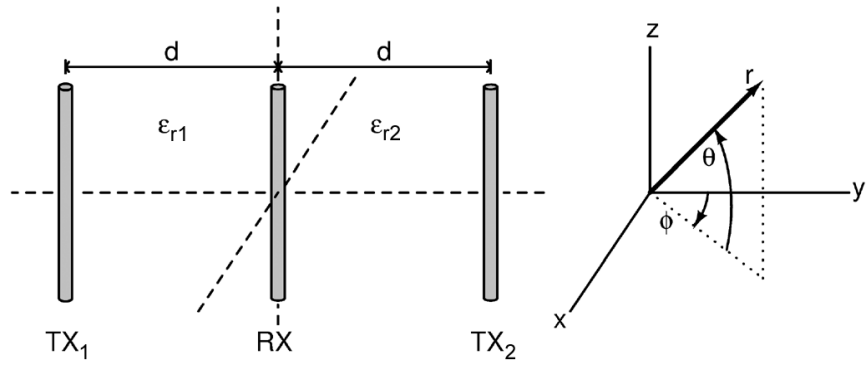

Fig. 1. Simplified view of the setup (the ground plane has been omitted for clarity). The figure shows two antennas $\left(\mathrm{TX}_{1}\right.$ and $\left.\mathrm{TX}_{2}\right)$ representing the transmit antenna (TX) and one antenna representing the receive antenna (RX).

the approaches described in [5] lead to a compromise between antenna efficiency and coupling reduction, and the approach in [6] leads to an increase of the receiver noise floor. A comparable passive principle described in [7] becomes challenging in a changing environment. In this letter, a broadband method of antenna coupling reduction is investigated without these compromises, but at the cost of adding one additional antenna. Using this principle, a receiver can effectively be isolated from a transmitter, possibly of different standards.

\section{ANALYSIS}

In Fig. 1, the setup is shown. In the analysis presented in this section, mutual coupling between the antennas is neglected to demonstrate the concept. Furthermore, the dependence on the elevation is not considered $(\theta=0)$, and the permeability is assumed to be equal to the value for free space $\left(\mu=\mu_{0}\right)$.

\section{A. Coupling}

Two transmit antennas $\left(\mathrm{TX}_{1}\right.$ and $\left.\mathrm{TX}_{2}\right)$ are placed with a distance $2 d$. Both transmit the same signal, but in opposite phase. In between these antennas, the receive antenna (RX) is placed. Because of the symmetry and opposite excitation phases, the RX antenna is isolated from the TX antennas.

The symmetry can be disturbed by the presence of a material in close proximity to the structure. As a result, the electromagnetic properties on the one side will be different than the electromagnetic properties on the other side, leading to a deviation from the wanted phase and magnitude to achieve full isolation. Modeling of this effect is done using the following equation, with $E_{\mathrm{TX}_{r}}=\gamma_{x}\left(E_{\mathrm{TX}} / 2\right)$ equal to the electric field at antenna RX due to $\mathrm{TX}_{x}$. The single side loss is modeled by $0 \leq$ $\gamma_{x} \leq 1$. The wavenumber $\beta_{x}$ is equal to $2 \pi / \lambda_{x}=\omega \sqrt{\epsilon_{x} \mu_{0}}$. The coupling is then given by

$$
\frac{\left|E_{\mathrm{RX}}\right|^{2}}{\left|E_{\mathrm{TX}}\right|^{2}}=\frac{\gamma_{1}^{2}+\gamma_{2}^{2}-2 \gamma_{1} \gamma_{2} \cos \left(\beta_{1} d-\beta_{2} d\right)}{4} .
$$




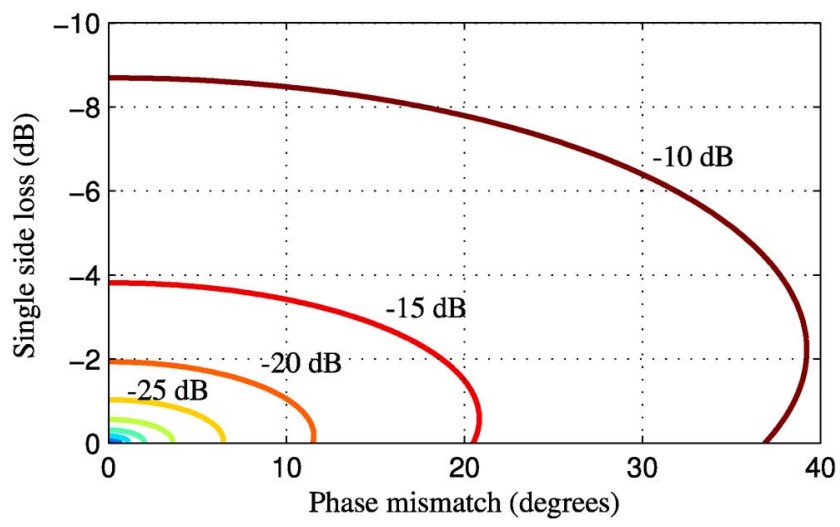

Fig. 2. Contour plot showing the coupling as a function of the single side loss and phase mismatch. The contours indicate a decrease of coupling by $5 \mathrm{~dB}$. The coupling is seen to be symmetric concerning the phase mismatch.

As can be seen in (1), the coupling reduces to zero when both $\gamma_{1}=\gamma_{2}$ and the following equations holds:

$$
\beta_{1}-\beta_{2}=\frac{2 k \pi}{d}, \quad k \in \mathbb{N}_{0} .
$$

In many realistic situations the environment continuously changes, resulting in a changing $\beta_{x}$ and $\gamma_{x}$. This can, for example, be caused by the presence of a hand/head in the neighborhood of the antenna. To guarantee low coupling between the antennas, the phase and/or magnitude difference between the two TX antennas should be continuously adapted. Identifying $\left(\beta_{1} d-\beta_{2} d\right)$ in (1) as a phase mismatch from the ideal phase to achieve isolation, the coupling as a function of phase mismatch and loss $\gamma_{1}$ is shown in Fig. $2\left(\gamma_{2}=1\right)$.

To achieve full isolation, $\gamma_{1}$ must be equal to $\gamma_{2}$, and an additional phase difference $\alpha$ between the two TX antennas must be included to guarantee $\left(\beta_{1} d-\beta_{2} d-\alpha\right)$ to be equal to $2 k \pi / d$. The equation for $\alpha$ is given as follows:

$$
\alpha=\frac{2 \pi d}{\lambda_{0}}\left[\sqrt{\epsilon_{\mathrm{r}, 1}}-\sqrt{\epsilon_{\mathrm{r}, 2}}\right]-\frac{2 k \pi}{d} .
$$

\section{B. Practical Implementation}

As discussed in Section I, it might be needed to continuously adapt the phase and magnitude difference of the outer antennas to guarantee low coupling. In Fig. 3, a possible implementation of this is proposed, using a transformer with multiple taps to divide the transmit power between the antennas in discrete steps. Equation (3) shows that the needed phase difference is inversely proportional to $\lambda$ and can be identified as a time delay, guaranteeing broadband operation. These time delays should behave with high linearity because of the strong signals processed. Therefore, in Fig. 3, adjustable time delays using microelectromechanical systems (MEMS) are suggested. If the time delay is adjusted using discrete steps, which is more practical, it can be seen that the maximum amount of coupling is equal to

$$
\left.\frac{\left|E_{\mathrm{RX}}\right|^{2}}{\left|E_{\mathrm{TX}}\right|^{2}}\right|_{\max }=\sin ^{2}\left(\frac{\text { Phase step }}{4}\right) \text {. }
$$

The number of phase steps needed to guarantee the desired coupling reduction depends on the maximum expected relative permittivity $\epsilon_{\mathrm{r}}$ [see (3)]. The relative permittivity in the presence

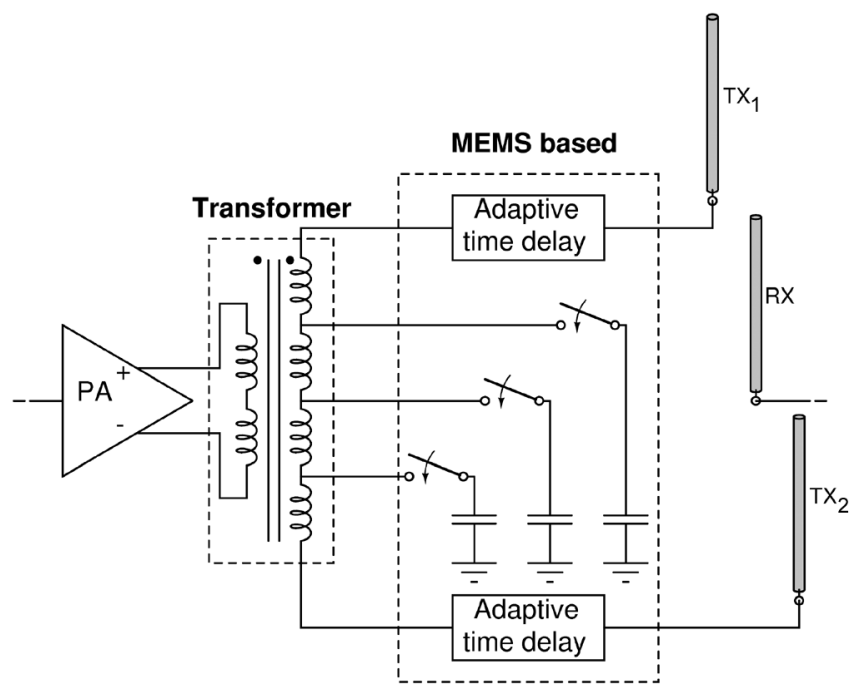

Fig. 3. Possible implementation of the antenna cancellation using different time delays in combination with a transformer with various taps.

of the human body [8] can reach high values, depending on the frequency and body part. The number of steps can be minimized by assuring small differences between $\epsilon_{\mathrm{r}, 1}$ and $\epsilon_{\mathrm{r}, 2}$. When the entire structure is put in an environment with a high $\epsilon_{\mathrm{r}}$, this is achieved. The entire structure then, of course, has to shrink to keep comparable electromagnetic properties, which is also a desirable property, especially in handheld devices. Although this is not trivial, comparable methods are applied in existing commercial devices (e.g., Bluetooth antennas).

\section{Radiation Pattern}

The close proximity of the antennas has an effect on the farfield behavior as well. Again, the elevation angle $\theta$ is kept equal to zero. Because of the setup, the radiation pattern of the outer two antennas is not influenced by the middle antenna. The electric field can be calculated by the following equations, using a differential excitation of the two outer antennas (with $E_{1}$ and $E_{\mathrm{r}}$ being the electric fields at the outer antennas):

$$
\begin{aligned}
E & =E_{1} \frac{e^{j\left(\omega t-\beta r_{1}\right)}}{r_{1}}+E_{\mathrm{r}} \frac{e^{j\left(\omega t-\beta r_{2}+\pi\right)}}{r_{2}} \\
r_{1} & =\sqrt{r^{2}+d^{2}+2 r d \cos \phi} \\
r_{2} & =\sqrt{r^{2}+d^{2}-2 r d \cos \phi} .
\end{aligned}
$$

The radiation pattern of the middle antenna will also be affected by the setup. Depending on the common-mode loading of the outer two antennas, the far electric field will be as follows (with $c$ a complex constant representing the interaction between the outer antennas and the middle antenna, and $E_{\mathrm{m}}$ the electric field generated at the middle antenna):

$$
\begin{aligned}
E= & E_{\mathrm{m}} \frac{e^{j(\omega t-\beta r)}}{r}+c \cdot E_{\mathrm{m}} \frac{e^{j\left(\omega t-\beta\left(r_{1}+d\right)\right)}}{r_{1}} \\
& +c \cdot E_{\mathrm{m}} \frac{e^{j\left(\omega t-\beta\left(r_{2}+d\right)\right)}}{2} .
\end{aligned}
$$

\section{Simulations AND MeAsurements}

Several simulations and measurements have been performed to verify the behavior. For this purpose, some prototypes have 


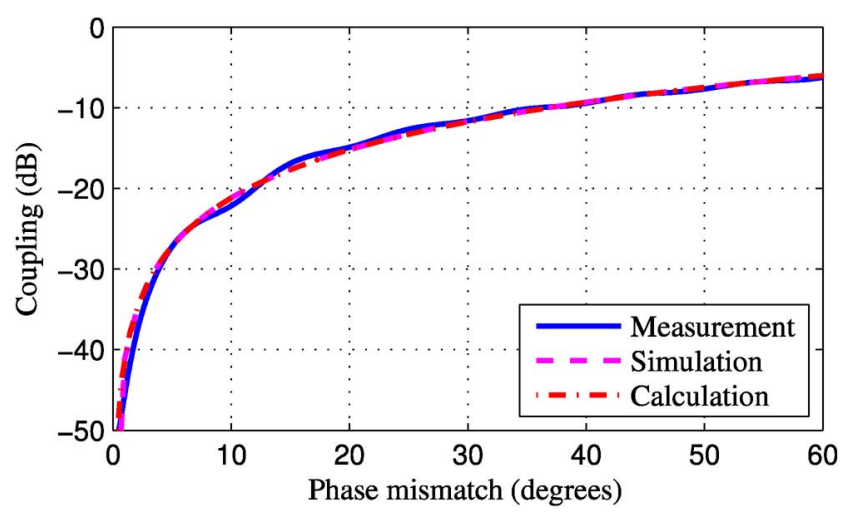

Fig. 4. Calculated, simulated, and measured coupling for varying phase difference in degrees. The simulation and measurement results have been corrected for the loss observed at a phase mismatch of $180^{\circ}$.

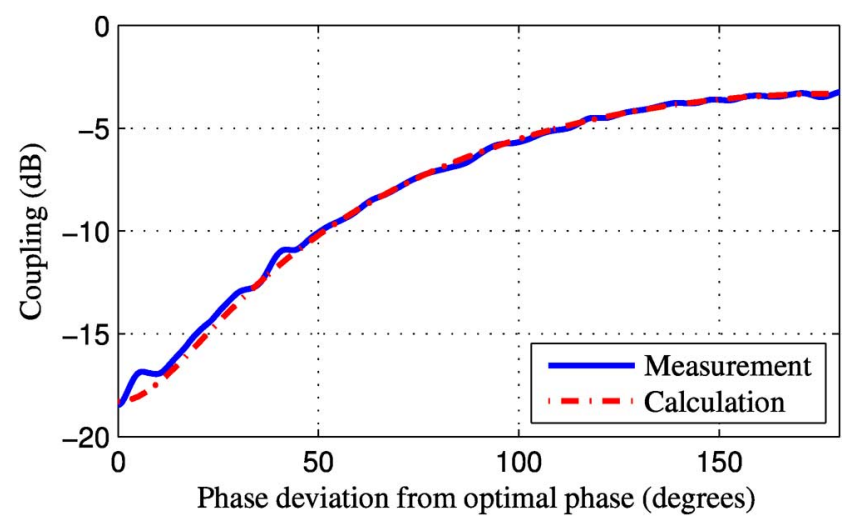

Fig. 5. Calculated and measured coupling for varying phase difference in degrees. The environment of the antenna structure has been made asymmetrical using salt water. The simulation and measurement results have been corrected for the loss observed at a phase mismatch of $180^{\circ}$.

been constructed consisting of three monopoles above a ground plane. The dimensions of the antennas were chosen to be resonant at $2.5 \mathrm{GHz}$ (length approximately $28 \mathrm{~mm}$ ). Prototypes have been constructed with different spacings $d$.

\section{A. Coupling}

As can be seen in Fig. 4, the calculated, simulated, and measured results of the coupling versus phase mismatch show good resemblance. The performed measurements show a coupling reduction of at least $50 \mathrm{~dB}$. This bound is encountered due to limited measurement accuracy.

By approaching the structure by salt water in an asymmetric fashion, the environment changes. In Fig. 5, the measured and calculated result is shown in such a situation. The horizontal axis shows the phase deviation from the optimal phase (to achieve lowest coupling). The minimal coupling before adjusting the power division to both antennas in this case increases to $-18 \mathrm{~dB}$. Measurements show that by dividing the power to both antennas in the right fashion, the coupling again reduces to the performance shown in Fig. 4.

\section{B. Impedance}

The impedance of the antenna structures have been measured using a network analyzer. This measurement was performed

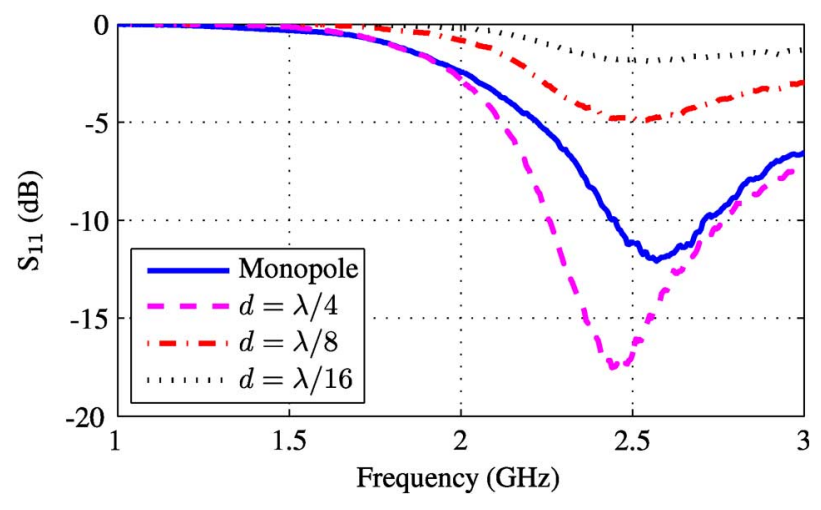

Fig. 6. $S_{11}$ of the outer antennas excited differentially with different spacings $d$ and, as a reference, the $S_{11}$ of a single monopole antenna. The spacing between the antennas is equal to $2 d$ (see Fig. 1 ).

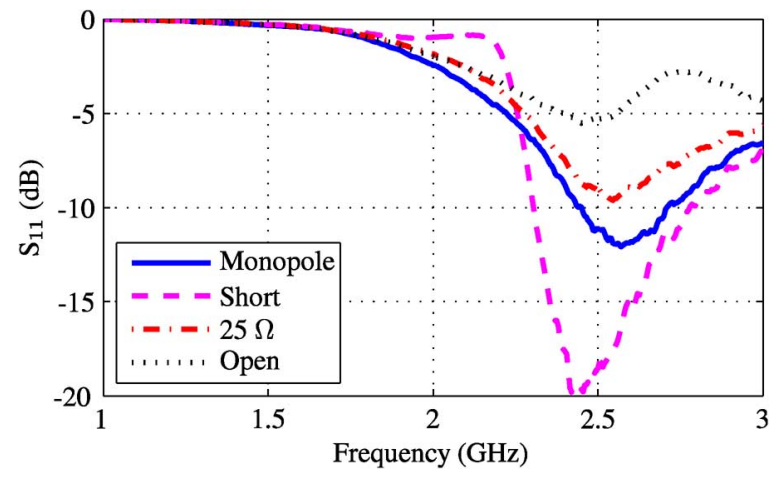

Fig. 7. $S_{11}$ of the middle antenna with different (common mode) loadings of the outer antennas $\left(d=\lambda / 4\right.$ and, as a reference, the $S_{11}$ of a single monopole antenna.

for both the outer (differential) antennas as well as for the middle antenna. Because of the coupling reduction from the outer antennas to the middle antenna, the impedance of the outer antennas is not influenced by the loading of the middle antenna. Measurements have been performed to verify this, connecting an open, short, or $50-\Omega$ load to the middle antenna. The impedance does depend on the spacing between the antennas. The measured $S_{11}$ for various values of $d$ confirm this. It is seen in Fig. 6 that the best performance is achieved using a space of $\lambda / 2$ between the antennas $(d=\lambda / 4)$.

The impedance of the middle antenna is also heavily influenced by the presence of the outer antennas. Because the best results concerning impedance of the outer antennas is observed with $d=\lambda / 4$, this setup was chosen during the measurements of the impedance of the middle antenna. The only degree of freedom we have left now is the common-mode loading of the outer antennas because the differential loading does not influence the impedance of the middle antenna. The latter was verified using measurements. The outer antennas were loaded with a short, open, and $25-\Omega$ resistor ( $50 \Omega$ each). The result of this measurement is shown in Fig. 7. From this, it can be concluded that the best performance is observed by using a shorted common-mode response. This can be achieved by using capacitors in series with the taps of the transformer, as shown in Fig. 3. These capacitors should be chosen such that it tunes out the inductance of the transformer, which will result in the required short for common-mode signals. 


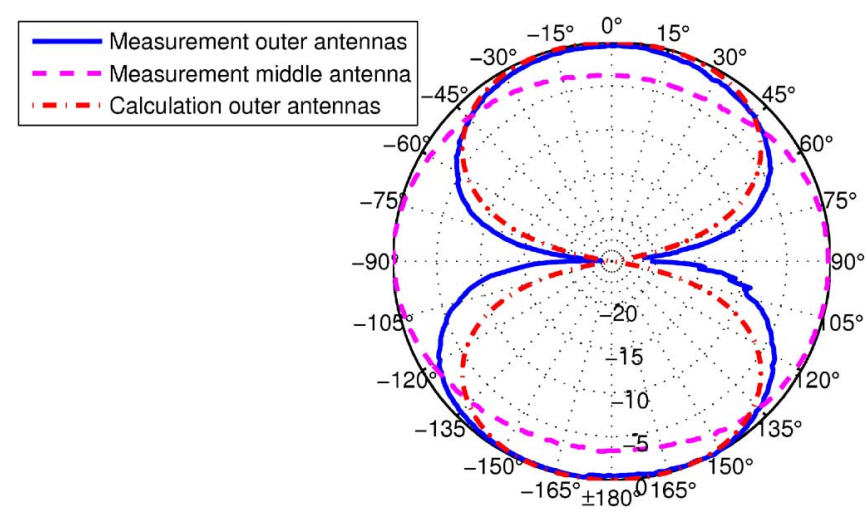

Fig. 8. Azimuth radiation pattern in decibels of the outer antennas (measurement and calculation) and of the middle antenna (measurement). The maximum antenna gain is normalized to $0 \mathrm{~dB}$.
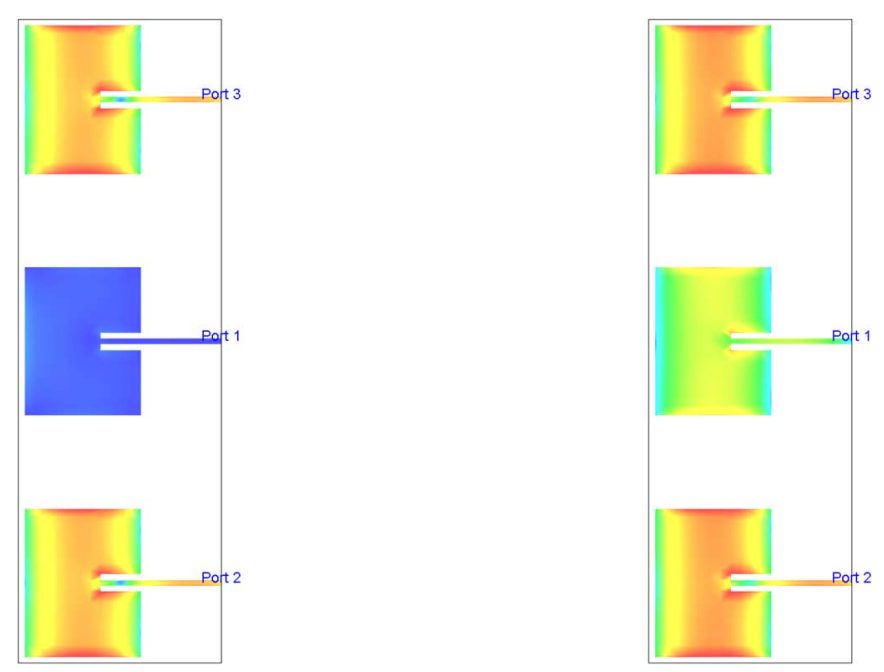

Fig. 9. Two current density plots of a simulation level implementation using patch antennas. The left figure shows the result using a differential excitation of the outer antennas, and the right using a common excitation. In the case of the differential excitation, no current is induced in the middle antenna, and in the case of the common excitation, there is current induced.

\section{Radiation Pattern}

The radiation patterns were measured for both antennas at $2.5 \mathrm{GHz}$. Measurements were performed in azimuth $(\phi)$, again with elevation angle $\theta=0$. In Fig. 8, these radiation patterns are shown. It is seen that a notch is created in the pattern of the outer antennas at $\phi=+/-90^{\circ}$. This can be understood by looking at (5)-(7). In this situation, $r_{1}=r_{2}$, resulting in combination with the $180^{\circ}$ phase difference between the two antennas in the observed notch. This is a fundamental property of this setup. However, in applications where there is a multipath effect, this should not be an issue. In general, multipath effects are omnipresent for many applications thanks to the presence of, e.g., objects in the environment and buildings.

Also, the radiation pattern of the middle antenna is shown with the outer antennas shorted to ground at their feed (achieving good impedance match). A slight deformation with respect to the pattern of a monopole antenna without obstructions in its neighborhood is observed.

For further verification, electromagnetic (EM) simulations were performed on patch antennas as well (see Fig. 9, ADS
Momentum). Results showed expected behavior concerning radiation patterns and impedance, as well as coupling behavior as described by (1).

\section{CONCLUSION}

In this letter, a method is discussed to decrease the coupling between a receive antenna in close proximity of a transmit antenna, enabling the simultaneous operation of a receiver and a transmitter of different standards. Coupling reduction between these two antennas is caused by the creation of a spatial notch at the position of the receive antenna by adding an additional antenna. The application of this concept has implications concerning the antenna pattern of mainly the transmit antenna. However, in an environment with multipath effects, this should not be an issue.

The structure has been verified using simulations and measurements on a structure based on monopole antennas, which show good resemblance with the analytical results. The amount of coupling reduction depends on how accurate the phase and magnitude in the setup can be matched to the desired values. Measurements have shown a coupling reduction of at least 50 $\mathrm{dB}$. With respect to the impedance of the differential excited antenna as well as with the middle antenna, satisfying results were obtained ( $S_{11}$ better than $-15 \mathrm{~dB}$ at frequency of interest). Additional EM simulations have also been performed using patch antennas, confirming the described behavior.

The application of this principle to a multimode transceiver promises the isolation between an active transmitter and receiver. It achieves this without decreasing the efficiency or signal-to-noise ratio. Furthermore, the dynamic range of the RF front end does not have to be increased. Additional cost that does come with this approach is an investment in size.

\section{ACKNOWLEDGMENT}

The authors would like to thank $H$. ten Pierick and H. van der Ploeg for their fruitful discussions and A. Reniers for his help during the measurements.

\section{REFERENCES}

[1] J. Zhu, A. Waltho, X. Yang, and X. Guo, "Multi-radio coexistence: Challenges and opportunities," in Proc. 16th IEEE Int. Conf. Commun. Netw., Aug. 2007, pp. 358-364.

[2] A. Palin and M. Honkanen, "VoIP call over WLAN with Bluetooth headset multiradio interoperability solutions," in Proc. Int. Symp. Pers., Indoor Mobile Radio Commun., Sep. 2005, vol. 3, pp. 1560-1564.

[3] B. Xavier and L. Christensen, "Cellular/WiFi multi-mode systems," in Proc. IEEE Radio Wireless Symp., 2006, pp. 3-6.

[4] Y. Xu, K. Wang, T. Pals, A. Hadjichristos, K. Sahota, and C. Persico, "A low-IF CMOS simultaneous GPS receiver integrated in a multimode transceiver," in Proc. IEEE Custom Integr. Circuits Conf., Sep. 2007, pp. 107-110.

[5] S. V. Georgakopoulos, "Coupling reduction for collocated antennas on MIMO systems," in Proc. 4th IEEE Int. Conf. Wireless Mobile Commun., Jul. 2008, pp. 325-330.

[6] A. Raghavan, S. Chandramouli, E. Gebara, and J. Laskar, "A low additive noise interference canceller for high sensitivity applications," in Proc. IEEE Radio Wireless Symp., Orlando, FL, Jan. 2008, pp. 45-48.

[7] D. Esser, B. Solan, and H. Chaloupka, "Improved antenna isolation in transmit/receive applications," presented at the GeMiC, 2006.

[8] A. Christ, T. Samaras, A. Klingenböck, and N. Kuster, "Characterization of the electromagnetic near-field absorption in layered biological tissue in the frequency range from $30 \mathrm{MHz}$ to $6000 \mathrm{MHz}$," Phys. Med. Biol., vol. 51, no. 19, pp. 4951-4965, Oct. 2006. 